\title{
Comparison of Automated System Vitek-2 with Conventional Methods, for Identification and Antibiotic Sensitivity in Gram Positive Organisms.
}

\author{
Nayeem u din Wani, Nargis Bali, Lenah Bashir, Junaid Ahmad, Suhail Ahmad
}

Department of Microbiology, SKIMS

\section{A B S T R A C T}

Background: Prompt identification (ID) and antimicrobial susceptibility testing (AST) of organisms causing blood stream infections has a significant impact on the morbidity and mortality associated with these infections. The need to circumvent the slow turnaround time of conventional gold standard methods has paved way for the rapid automated systems.

Aim: To compare the results of Vitek-2 for the ID andAST of Gram positive isolates with conventional manual methods.

Material and Methods: A total of 215 non-duplicate isolates of Gram positive bacteria recovered from blood samples were part of this prospective study carried out in the Department of Microbiology. Organisms were processed on the Vitek-2 system and by manual methods (ID/AST) for comparison. Descriptive statistics was used for the presentation and comparison of data and appropriate statistical charts were used to present the data.

Observations: Concordant identification (ID) results of Vitek-2 were seen with all the isolates of $S$. aureus, $S$. epidermidis, S. pneumonia, $E$. faecalisand $E$. faecium. Discordant results of Vitek-2 were seen for $S$. hominis ( 5 isolates of the organism misidentified as $S$. epidermidis). No minor, major or very major error with $100 \%$ categorical agreement $(C A)$ was seen for penicillin, cefoxitin, oxacillin, linezolid, ciprofloxacin, tetracycline, co-trimoxazole, clindamycin and erythromycin for various organisms tested. Enterococci gave minor error of $4.5 \%$ with an overall CA of $95.5 \%$ for ampicillin and a major error of $2.8 \%$ with an overall CA of $97.2 \%$ for vancomycin.

Conclusion: The organisms having slow metabolic rates and late lactose fermenters (S. hominis) are prone to errors by the Vitek-2 system; hence need to be reconfirmed with other possible method. Also AST results for critical antibiotics like vancomycin need to be verified manually before reporting.

Key words: Antimicrobial Susceptibility testing, Enterococci, Vitek-2

JMS: 2019; 22(2):12-17 DOI: https://doi.org/10.33883/jms.v22i2.433

\section{INTRODUCTION}

Infectious diseases account for two million deaths in India each year. ${ }^{1}$ Clinical microbiology laboratory performs identification (ID) and antimicrobial susceptibility testing (AST) of significant bacterial isolates with the aim to guide antibiotic therapy and thus reduce the morbidity and mortality associated with infectious diseases.Rapid bacterial identification and susceptibility testing apart from improving patient therapy and outcome, decreases the emergence of resistance and also reduces costs. Although considered as the gold standard conventional methods for

\begin{tabular}{l|l} 
Access this article online \\
Website: \\
WoI:.jmsskims.org
\end{tabular}

the ID and AST suffer from inherent drawbacks such as the slow turn-around time. The needs of the hour are rapid methods that can determine antibiotic susceptibility and enable targeted treatment as early as possible. Automated ID/AST systems can aid in rapid diagnosis/treatment of bacterial pathogens and have been in the market for many

Correspondence:

Dr. Nayeem u din Wani, MD

Senior Resident, Department of Microbiology, SKIMS

Email:drnayeemwani@gmail.com

How to cite this article: Wani N, Bali N, Bashir L, Ahmad J, Ahmad S. Comparison of Automated System Vitek-2 with Conventional Methods, for Identification and Antibiotic Sensitivity in Gram Positive Organisms. jms 2019;22(2):12-7

Received: 20-03-2019 Accepted: 15-05-2019 
Wani N; et al; Comparison of Automated System Vitek-2 with Conventional Methods,

years now. ${ }^{3,4}$

Although automation is justified for chemistry and hematology laboratories, the relative specimen and test volumes for microbiology are much smaller, making automation seemingly less attractive in this field. ${ }^{5}$ Nonetheless a reduction in labor, faster reporting of results especially in blood stream infections and identification of uncommon or rare organisms are some of the reasons as to why automation in Microbiology has gained popularity in recent years. ${ }^{6}$ The current study was thus designed to compare automated ID and AST system for Gram positive isolates with conventional manual methods in our laboratory.

\section{MATERIAL AND METHODS}

This prospective study was conducted in the Department of Clinical Microbiology at a leading tertiary care institute of North India from August 2016 to January 2017. Bar coded blood culture bottles received from different areas of hospital from patients suspected of having a blood stream infection were loaded into the BacT/Alert Microbial Detection System. Flag positive bottles were taken out and cultured on to blood agar and Mac-Conkey agar. Inoculated plates were incubated at $37^{\circ} \mathrm{C}$ overnight. Next day growth was inspected and Gram staining done to confirm the findings. Only Gram positive isolates were included in this study.

Inoculums were prepared for processing on to the Vitek-2 system for ID and AST as per the standard Vitek-2 inoculation procedure using the ID and AST cards. The Vitek-2 system reported the results automatically with software release 2.01. Part of the same specimen was processed by conventional manual methods for subsequent comparison. Manual identification was based on routine spot tests and standard biochemical reactions whereas antibiotic sensitivity testing (AST) was done by Kirby Bauer disc diffusion method, according to Clinical Laboratory Standard Institute (CLSI) guidelines. ${ }^{7}$ AST by Microbroth dilution was done with those antibiotics for which the interpretative criterion is based on MIC values only. Results of Vitek-2 system were compared with results obtained from conventional methods.

Terms used in the study: The "consistent ID" category implied that bacteria were equally identified at the genus and species level by both the conventional and the Vitek-2 systems. In the event of discordant ID results, the assay was repeated with both systems. Results of ID tests obtained with the conventional system were used as a reference. Antimicrobial susceptibility results were expressed as raw data. Results of susceptibility tests were categorized as susceptible (S), intermediate (I) or resistant (R) according to criteria recommended by CLSI. ${ }^{7}$

Interpretative values obtained by Vitek- 2 were compared to those obtained with the reference methods. The following definitions were adopted:

1) Essential agreement (MIC values of test panel equal to or within 1 dilution of the reference value).

2) Categorical agreement (Test and reference MIC values agree using the interpretative CLSI criteria).

3) Minor errors (Reference is $\mathrm{S}$ or $\mathrm{R}$ and test is $\mathrm{I}$; alternatively, reference is I and test is $\mathrm{S}$ or R).

4) Major errors (Reference is $\mathrm{S}$ and test is $\mathrm{R}$ ); the percentage of major errors was calculated only for susceptible isolates.

5) Very major errors (Reference is $\mathrm{R}$ and test is $\mathrm{S}$ ); the percentage of very major errors was calculated only for resistant isolates.

\section{Statistical Analysis}

Descriptive statistics was used for the analysis and comparison of data and appropriate statistical charts were used to present the data.

\section{Observations}

A total of 215 non-duplicate isolates of Gram positive bacteria recovered from blood samples were included in the study. Majority of samples received belonged to male patients 53.5\% $(n=115)$ and $46.5 \%(n=100)$ were from female patients. Also most of the samples were received from patients in the age group of $60-69$ years, $27 \%(n=58)$ followed by $40-49$ years, $16.7 \%(n=36) ; 50-59$ years, $14.9 \%$ $(n=32)$ and 30-39 years, 12.1\% $(n=26)$ whereas least number of samples belong to patients in the age group of 20-29 years, $8.8 \%(n=19) ; 10-19$ and $>70$ years, $7.4 \%(n=16$ each) and 0-9 years, 5.6\% $(\mathrm{n}=12)$. Most of the samples came from patients admitted in the General Medicine ward, 13\% $(\mathrm{n}=28)$, Surgical Intensive Care Unit, 12.1\% $(\mathrm{n}=26)$ and General Surgery, $11.2 \%$ (24). The isolation of samples according to the age, gender and ward was not statistically 
Wani N; et al; Comparison of Automated System Vitek-2 with Conventional Methods,

significant.

Most common Gram positive isolates recovered were Staphylococcus aureus $21.8 \%(\mathrm{n}=47)$ followed by Enterooccusfeacalis $19 \% \quad(\mathrm{n}=41)$, Staphylococcus epidermidis 17.2\% ( $\mathrm{n}=37)$, Enterooccusfaecium $16.7 \%$ $(\mathrm{n}=36)$ Staphylococcushaemolyticus $14.4 \%$ $(\mathrm{n}=31)$ Staphylococcushominis $9.7 \% \quad(\mathrm{n}=21)$ and Streptococcus pneumoniae $0.9 \% \quad(\mathrm{n}=2)$. Concordancebetween the results obtained from Vitek-2 and manual ID was seen with all the isolates of $S$. aureus, $S$. epidermidis, S. pneumonia, E. faecalisand E. faecium. However discordant results were obtained for $S$. hominis, as five isolates of the organism were miss-identified as $S$. epidermidis by Vitek-2. Table 1

Table 1: Identification of organisms by Vitek-2 and comparison with conventional methods

\begin{tabular}{|c|c|c|c|}
\hline \multirow[t]{2}{*}{ Organism } & \multirow{2}{*}{$\begin{array}{l}\text { No of } \\
\text { isolates } \\
\text { tested }\end{array}$} & \multicolumn{2}{|c|}{ No. (\%) of ID's } \\
\hline & & Concordant & Discordant \\
\hline S. aureus & 47 & $47(100 \%)$ & 0 \\
\hline S. epidermidis & 37 & $37(100 \%)$ & 0 \\
\hline E. faecalis & 41 & $41(100 \%)$ & 0 \\
\hline E. faecium & 36 & $36(100 \%)$ & 0 \\
\hline S. haemolyticus & 31 & $31(100 \%)$ & 0 \\
\hline S. hominis & 21 & $16(76.2 \%)$ & $5(23.8 \%)$ \\
\hline S. pneumoniae & 2 & $2(100 \%)$ & 0 \\
\hline Total & 215 & $210(97.7 \%)$ & $5(2.3 \%)$ \\
\hline
\end{tabular}

Antibiotic susceptibility results of all drug classes as given by Vitek-2 and reference method are shown in table 2. No minor, major or very major errors with $100 \%$ categorical agreement (CA) was seen for penicillin, cefoxitin, oxacillin, linezolid, ciprofloxacin, tetracycline, co-trimoxazole, clindamycin and erythromycin against the organisms tested. However Enterococci gave minor error of $4.5 \%$ with an overall CA of $95.5 \%$ for ampicillin and major error of $2.8 \%$ with an overall CA of $97.2 \%$ for vancomycin. No very major errors were seen for these antibiotics. Table 3

\section{DISCUSSION}

A total of 215 non-duplicate isolates of Gram positive bacteria were part of the present study. Specimens were received more from male patients in our study, similar to what was reported by Nadheema et al. ${ }^{8}$ Highest culture positivity was seen in samples received from General medicine, SICU and General surgery ward. This observation is in accordance to what was seen by Maria et $a l$. who reported that majority of samples were from patients housed in medical and associated intensive care units. ${ }^{9} S$. aureus followed by S. epidermidis were the most common Gram positive bacteria isolated in our study. Similar results were seen in a study done by de Cueto $\mathrm{M}$ et al. where the most common Gram positive cocci were reported to be $S$. aureus and S. epidermidis. ${ }^{10}$

In this study concordance was seen between the ID results of

Table 2:Comparison of AST by the two methods.

\begin{tabular}{|l|l|l|l|l|l|l|l|}
\hline Gram positive bacteria & \multirow{2}{*}{ Total } & \multicolumn{2}{l}{ Vitek-2 N (\%) } & \multicolumn{3}{l|}{ Manual AST N (\%) } \\
\cline { 3 - 8 } & & S & R & I & S & R \\
\hline Penicillin & 215 & $6(2.8)$ & $209(97.2)$ & 0 & $6(2.8)$ & $209(97.2)$ \\
\hline Ampicillin & 77 & $26(33.7)$ & $47(61)$ & $4(5.1)$ & $36(46.8)$ & $41(53.2)$ \\
\hline Cefoxitin & 136 & $72(52.9)$ & $64(47.1)$ & 0 & $72(52.9)$ & $64(47.1)$ & 0 \\
\hline Oxacillin & 2 & $2(100)$ & 0 & 0 & $2(100)$ & 0 & 0 \\
\hline Vancomycin & 215 & $201(93.5)$ & $14(6.5)$ & 0 & $207(96.3)$ & $8(3.7)$ \\
\hline Linezolid & 215 & $207(96.3)$ & $8(3.7)$ & 0 & $207(96.3)$ & $8(3.7)$ \\
\hline Ciprofloxacin & 215 & $147(68.3)$ & $68(31.6)$ & 0 & $147(68.4)$ & $68(31.6)$ \\
\hline Tetracycline & 148 & $66(44.6)$ & $82(55.4)$ & 0 & $66(44.6)$ & $82(55.4)$ \\
\hline Co-trimoxazole & 138 & $67(48.5)$ & $71(51.4)$ & 0 & $67(48.5)$ & $71(51.4)$ \\
\hline Clindamycin & 138 & $78(56.5)$ & $60(43.4)$ & 0 & $78(56.5)$ & $60(43.4)$ \\
\hline Erythromycin & 138 & $37(26.8)$ & $101(73.1)$ & 0 & $37(26.8)$ & $101(73.1)$ \\
\hline
\end{tabular}


Wani N; et al; Comparison of Automated System Vitek-2 with Conventional Methods,

Table 3: Organism vise susceptibility to antimicrobials.

\begin{tabular}{|l|l|l|l|l|l|l|l|l|l|l|l|l|l|}
\hline \% susceptibility & \multicolumn{2}{l}{ Staphylococci(n=136) } & \multicolumn{2}{l|}{ Enterococci(n=77) } \\
\hline Antimicrobial & CA & mE & ME & VME & CA & mE & ME & VME & CA & mE & ME & VME \\
\hline & 100 & 0 & 0 & 0 & 100 & 0 & 0 & 0 & 100 & 0 & 0 & 0 \\
\hline Penicillin & - & - & - & - & 95.5 & 4.5 & 0 & 0 & - & - & - & - \\
\hline Ampicillin & 100 & 0 & 0 & 0 & - & - & - & - & - & - & - & - \\
\hline Cefoxitin & - & - & - & - & - & - & - & - & 100 & 0 & 0 & 0 \\
\hline Oxacillin & 100 & 0 & 0 & 0 & 97.2 & 0 & 2.8 & 0 & 100 & 0 & 0 & 0 \\
\hline Vancomycin & 100 & 0 & 0 & 0 & 100 & 0 & 0 & 0 & 100 & 0 & 0 \\
\hline Linezolid & 100 & 0 & 0 & 0 & 100 & 0 & 0 & 0 & 100 & 0 & 0 \\
\hline Ciprofloxacin & 100 & 0 & 0 & 0 & 100 & 0 & 0 & 0 & 100 & 0 & 0 \\
\hline Tetracycline & 100 & 0 & 0 & 0 & - & - & - & - & 100 & 0 & 0 \\
\hline Co-trimoxazole & 100 & 0 & 0 & 0 & - & - & - & - & 100 & 0 & 0 \\
\hline Clindamycin & 100 & 0 & 0 & 0 & - & - & - & - & 100 & 0 & 0 \\
\hline Erythromycin & & & & & & & & 0 \\
\hline
\end{tabular}

CA: Categorical agreement; mE: minor error; ME: major error; VME: very major erro

Vitek-2 and the manual methods for all isolates of S. aureus, S. hemolyticus, S. pneumonia, E. faecalisand E. faecium. However, discordant results were seen for $S$. hominis wherein 5 isolates were wrongly identified as $S$. epidermidis by the instrument. Spanuet al.in their study found that 3 out of 7 isolates of $S$. hominis were misidentified by the Vitek-2 system. The authors hypothesized that the inferior performance of this system viz avizCoagulase-negative Staphylococci might be related to the relatively low rates of metabolism displayed by these species. The possibility of false-negative reactions within the short incubation times used by this system may well have contributed to the lowdiscrimination results as $S$. hominis produces acid from lactose however the failure to do so in the Vitek-2 system due to short incubation period may have contributed to its misidentification. ${ }^{11}$ Fahr et al. studied 469 Gram positive isolates and found that Staphylococci, Enterococci, and Streptococci showed a concordant result in 97.1, 98.9, and $100 \%$ of cases, respectively. For one isolate of S. hominis, the Vitek-2 comparator system gave a discordant result and wrongly identified the isolate as $S$. haemolyticus. ${ }^{12}$
For the antibiotic susceptibility results of Gram positive organisms in our study no minor, major or very major error, with $100 \%$ categorical agreement (CA) was seen with penicillin, cefoxitin, oxacillin, linezolid, ciprofloxacin, tetracycline, co-trimoxazole, clindamycin and erythromycin. Enterococci however gave minor error of $4.5 \%$ with an overall CA of $95.5 \%$ for ampicillin and a major error of $2.8 \%$ with an overall CA of $97.2 \%$ for vancomycin. Similar results were seen by Marco Ligozziet $a l$ who evaluated the Vitek-2 system for identification and antibiotic susceptibility testing of Gram-positive cocci. In their study Vitek-2 system gave 96\% correct CA, $0.82 \%$ very major errors, $0.17 \%$ major errors, and $2.7 \%$ minor errors. Microorganism-antibiotic combinations that gave very major errors were CoNS-erythromycin, CoNSoxacillin, Enterococci-teicoplanin, and Enterococcigentamicin. Major errors were observed for CoNS-oxacillin and S. agalactiae-tetracycline combinations. ${ }^{13}$

In another study by Garrote GF et al.the authors found percentage agreement for susceptibility and resistance to ampicillin, vancomycin, teicoplanin, gentamicin, and streptomycin as $93,95,97,97$, and $96 \%$, respectively. 
Wani N; et al; Comparison of Automated System Vitek-2 with Conventional Methods,

Among the vancomycin-resistant strains, the essential agreement was $93 \%$. The Vitek-2 system detected 93 of 99 resistant isolates. Two strains in which vancomycin resistance was not detected, species identifications were also incorrect. Very major error rate ranged from $4 \%$ for vancomycin to $1.3 \%$ for ampicillin, teicoplanin and gentamicin. Nine ampicillin-susceptible $E$. faecalis isolates were categorized as ampicillin resistant and a betalactamase positive E. faecalis strain and an ampicillinresistant beta-lactamase negative $E$. faecalis strain were not detected as resistant by the Vitek-2. ${ }^{14}$

\section{Summary and conclusion}

With regards to AST results, it has been recommended by CLSI that an overall category error rate of $<10 \%$ is a must for accepting the performance of susceptibility tests, with up to $3.0 \%$ major errors and up to $1.5 \%$ very major errors. In our study no very major errors were found in the AST of Gram positive isolates whereas $2.8 \%$ major errors and $4.5 \%$ minor errors were in seen case of Enterococci for vancomycin and ampicillin; two of the most critical drugs for blood stream infections caused by these organisms. The AST results in case of these isolates must always be confirmed by manual method so that the patients receive appropriate antibiotic therapy. Furthermore, appropriate incubation conditions and duration should be specified for organisms having slow metabolic rates and late lactose fermenters like $S$. hominis that are prone to be misidentified by the Vitek-2 system.

\section{DECLARATIONS}

Conflict of interest: None

Funding Sources: Self-funded from SKIMS

Written informed consent: $\mathrm{n} / \mathrm{a}$

\section{REFERENCES}

1. Durand, S. 'Executive SummaryThe Globalization Of Infectious Disease'. www.prcdc.org/files/ Infectious Disease. N.p., 2000. Print.

2. Ling T, Liu Z, Cheng A. Evaluation of the VITEK 2 System for Rapid Direct Identification and Susceptibility Testing of Gram-Negative Bacilli from Positive Blood Cultures. Journal of Clinical Microbiology. 2003; 41(10):4705-4707.

3. Duggal S, Gaind R, Tandon N, Deb M, Chugh T.
Comparison of an Automated System with Conventional Identification and Antimicrobial Susceptibility Testing. ISRN Microbiology. 2012; 2012:1-4.

4. Jossart M, Courcol R. Evaluation of an Automated System for Identification of Enterobacteriaceae and Non-fermenting Bacilli. European Journal of Clinical Microbiology \& Infectious Diseases. 1999; 18(12):902-907.

5. Bourbeau P, Ledeboer N. Automation in Clinical Microbiology. Journal of Clinical Microbiology. 2013; 51(6):1658-1665.

6. Doern, G. V., R. Vautour, M. Gaudet, and B. Levy. 1994. Clinical impact of rapid in vitro susceptibility and bacterial identification. J. Clin. Microbiol.32:1757-1762.

7. Clinical and Laboratory Standards Institute. Performance standards for antimicrobial susceptibility testing; eighteenth information supplement. 2015; M100-S

8. Nadheema H., Khetam H.R., Jumaah D. H. Frequency of Extended Spectrum Beta Lactamase producing Gram negative bacteria isolated from blood cultures at children hospital in Baghdad Volume : 4 | Issue : 1 | January 2015 ISSN No 22778179.

9. Espinar MJ, Rocha R, Ribeiro M, Gonçalves Rodrigues A, Pina-Vaz C. Extended-spectrum $\beta$ lactamases of Escherichia coli and Klebsiella pneumoniae screened by the VITEK 2 system. Journal of Medical Microbiology. 2011; 60(6):756-60.

10. De Cueto M, Ceballos E, Martinez-Martinez L, Perea E, Pascual A. Use of Positive Blood Cultures for Direct Identification and Susceptibility Testing with the Vitek 2 System. Journal of Clinical Microbiology. 2004; 42(8):3734-3738.

11. Spanu T, Sanguinetti M, Ciccaglione D, D'Inzeo T, Romano L, Leone F et al. Use of the VITEK 2 System for Rapid Identification of Clinical Isolates of Staphylococci from Bloodstream Infections. Journal of Clinical Microbiology. 2003; 41(9):4259-4263.

12. Fahr A, Eigner U, Armbrust M, Caganic A, Dettori G, Chezzi $\mathrm{C}$ et al. Two-Center Collaborative Evaluation of the Performance of the BD Phoenix Automated 
Wani N; et al; Comparison of Automated System Vitek-2 with Conventional Methods,

Microbiology System for Identification and Antimicrobial Susceptibility Testing of Enterococcus spp. and Staphylococcus spp. Journal of Clinical Microbiology. 2003;41(3):1135-1142.

13. Ligozzi M, Bernini C, Bonora M, de Fatima M, Zuliani J, Fontana R. Evaluation of the VITEK 2 System for Identification and Antimicrobial Susceptibility Testing of Medically Relevant Gram-Positive Cocci. Journal of
Clinical Microbiology. 2002; 40(5):1681-1686.

14. Garrote F G, Cercenado E,Bouza E. Evaluation of a New System, VITEK 2, for Identification and Antimicrobial Susceptibility Testing of Enterococci. J. ClinMicrobiol. 2000 Jun; 38(6): 2108-2111. 